\title{
MIT 口袋的波包展开
}

\author{
蔡 既 杨仲乐 刘连寿
}

(华中师范学院)

MIT 袋模型的拉氏量 ${ }^{[0}$ 是明显平移不变的, 然而它的球腔近似解却不具有这种不变性. 为了克服这一仭点, Donoghue 和 Johnson ${ }^{[2]}$ 建议对球腔近似解作波包展开, 并将这种波包展 开应用于质子衰变寿命的计算 ${ }^{[3]}$.

在文献 [4] 中,我们进一步发展了波包展开的思想,提出了用袋模型处理强子动力学过程 的一种普遍方法. 这个方法充分注意到引起囚禁的集体自由度对强子过程的影响, 恰当地实 现了口袋整体运动与内部运动的分离, 自动地得到了 4-动量守恒, 从而避免了迄今为止不少 作者 ${ }^{[s-7,3]}$ 在用袋模型讨论强子衰变时完全忽略反冲效应的粗粘作法.

以 $x$ 介子为例, 通常处理强子静态问题的中心在 $x_{0}$ 处的球腔近似解可以用动量本征态展 开

$$
\left|x\left(x_{0}\right)\right\rangle_{t=0}=\int \frac{d^{3} p}{2 E_{p}} \varphi_{x}(p) e^{-i p \cdot x_{0}}|x(p)\rangle_{t=0} ;
$$

反过来,动量本征态又可以表为中心处于不同位置的口袋的迭加

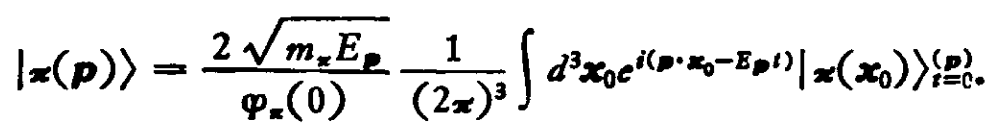

这是我们方法的基本公式. 显然, 在这一类波包展开方法中, 关键性的步骤在于确定波包函数 $\varphi(\boldsymbol{p})$ 和波包的中心值 $\varphi(0)$.

Donoghue 和 Johnson ${ }^{[2]}$ 计算 $x$ 介子的波包函数 $\varphi(p)$ 时, 利用了 $x \rightarrow \mu \nu$ 过程的流矩阵 元, 他们还假定这样求得的 $\varphi(\boldsymbol{p})$, 可以推广到一切介子和重子. 这种做法存在着两个严重的 缺点: 一是由于求得的 $\varphi(\boldsymbol{p})$ 依赖于计算矩阵元时所选用的流, 具有任意性, 使得 $\varphi(\boldsymbol{p})$ 不唯 一; 二是认为一切介子和重子都有相同形式的 $\varphi(\boldsymbol{P})$ ，缺乏根据. 产生这些缺点的原因是他们 的做法中间包含了一种非常粗楉的假定, 即忽略了不同的强子口袋的差别, 并认为空口袋和物 理真空等同.

为了消除 $\varphi(\boldsymbol{p})$ 的不唯一性,使得袋模型的波包展开方法具有可靠的基础，我们在本文中 提出了计算各种介子和重子波包函数的一种更为合理而普适的方法.

仍以 $x$ 介子为例, 中心在 $x_{0}$ 处的 $\pi$ 介子口袋态归一化为

$$
\left\langle x\left(x_{0}\right) \mid x\left(x_{0}\right)\right\rangle=1 \text {. }
$$

在强子过程中出现的动量本征态是中心在不同位置的口袋的迭加, 如式 (2). 因此, 有必要研 究中心在不同位置的口袋态的乘积 $\left\langle\boldsymbol{x}\left(\boldsymbol{x}_{0}^{\prime}\right) \mid \boldsymbol{x}\left(\boldsymbol{x}_{0}\right)\right\rangle$. 这一乘积只应依赖于两口袋中心的距离 $r=|\boldsymbol{r}|=\left|x_{0}^{\prime}-x_{0}\right|$, 因而令 


$$
\left\langle\boldsymbol{x}\left(\boldsymbol{x}_{\mathrm{r}}^{\prime}\right) \mid \boldsymbol{x}\left(\boldsymbol{x}_{0}\right)\right\rangle=F_{\mathrm{x}}(r),
$$

其中 $F_{x}(r)$ 应满足条件

$$
F_{x}(r)=\left\{\begin{array}{lll}
1 & \text { 当 } r=0, \\
0 & \text { 当 } r \geqslant 2 R_{x},
\end{array}\right.
$$

$R_{x}$ 为 $\pi$ 介子的口袋半径.

式 (4) 是归一化条件式 (3) 的推广. 为求 $F_{x}(r)$, 将二粒子(夸克和反夸克) 坐标本征矢 的完备性条件

$$
\int\left|x_{1} x_{2}\right\rangle\left\langle x_{1} x_{2}\right| d x_{1} d x_{2}=1
$$

插入 (4) 式的左边. 用 $u$ 和 $v$ 分别表示袋模型中正反夸克的球腔近似波函数 ${ }^{[5]}$, 则有(为简单 起见, 不明显写出波函数的 $S U(6)$ 和 $S U(3)_{c}$ 部分):

$$
\left\langle x_{1} x_{2} \mid \pi\left(x_{0}\right)\right\rangle=u\left(x_{1}-x_{0}\right) v\left(x_{2}-x_{0}\right),
$$

因而得到

$$
F_{x}(r)=\int_{Q} d^{3} x_{1} u^{+}\left(x_{1}-x_{0}^{\prime}\right) u\left(x_{1}-x_{0}\right) \int_{Q} d^{3} x_{2} v^{+}\left(x_{2}-x_{0}^{\prime}\right) v\left(x_{2}-x_{0}\right),
$$

其中 $\Omega$ 为两个口袋的重迭区域.

这一结果不难推广到其它介子和重子. 在重子情况下, 代替 (6) 式的是三粒子（三个夸 克)的坐标本征矢的完备性条件

$$
\int\left|x_{1} x_{2} x_{3}\right\rangle\left\langle x_{1} x_{2} x_{3}\right| d^{3} x_{1} d^{3} x_{2} d^{3} x_{3}=1 .
$$

在图 1 上画出了通过数值计算得到的介 子和重子的 $F(r)$ 曲线（以无因次变量 $x=$ $r / 2 R$ 为横坐标). 在同一图上还画出了忽略 强子内部的夸克分布、只考虑口袋重迭效应 而得到的近似公式

$$
\left\langle x\left(x_{0}^{\prime}\right) \mid \approx\left(x_{0}\right)\right\rangle \approx \frac{Q}{V_{z}} \equiv F_{\Omega}(r)
$$

的 $F_{Q}(r)$ 曲线

$$
F_{Q}(r)=\left\{\begin{aligned}
1-\frac{3}{4} \frac{r}{R_{x}} & +\frac{1}{16}\left(\frac{r}{R_{x}}\right)^{3}, \\
& \text { 当 } r \leqslant 2 R_{x}, \\
0, & \text { 当 } r>2 R_{x} .
\end{aligned}\right.
$$

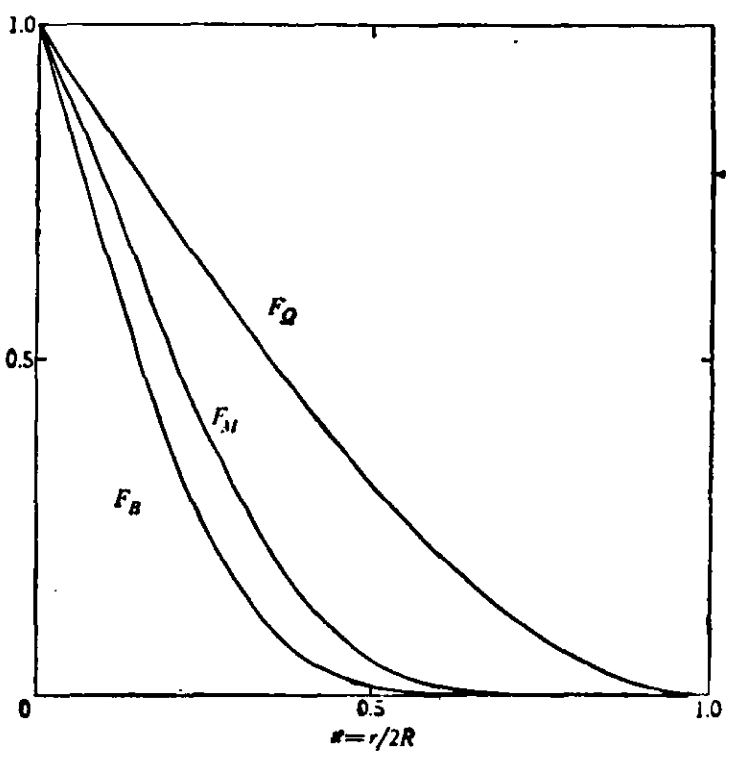

图 1

将波包展开式 (1) 代入 (4) 式,并利用动量本征态的正交归一性 $\left\langle\boldsymbol{x}\left(\boldsymbol{p}^{\prime}\right) \mid \boldsymbol{x}(\boldsymbol{p})\right\rangle=(2 \boldsymbol{x})^{3} \boldsymbol{\delta}^{3}$ $\cdot\left(\boldsymbol{p}^{\prime}-\boldsymbol{p}\right) 2 E_{\boldsymbol{m}}$, 立刻求出波包函数

$$
\left|\varphi_{x}(\boldsymbol{p})\right|^{2}=\frac{2 E_{\boldsymbol{x}}}{(2 x)^{6}} \int d^{3} r e^{-i p \cdot r} F_{x}(r) .
$$

这一公式适用于一切介子, 而对于重子有

$$
\left|\varphi_{B}(p)\right|^{2}=\frac{1}{(2 x)^{6}} \frac{E_{P}}{m_{B}} \int d^{3} r c^{-i \boldsymbol{r} \cdot r} F_{B}(r) .
$$

根据这两个公式, 利用图 1 的结果, 可求得 $x$ 介子、 $\rho$ 介子和质子的波包函数的模 $|\varphi(\boldsymbol{p})|$, 如 


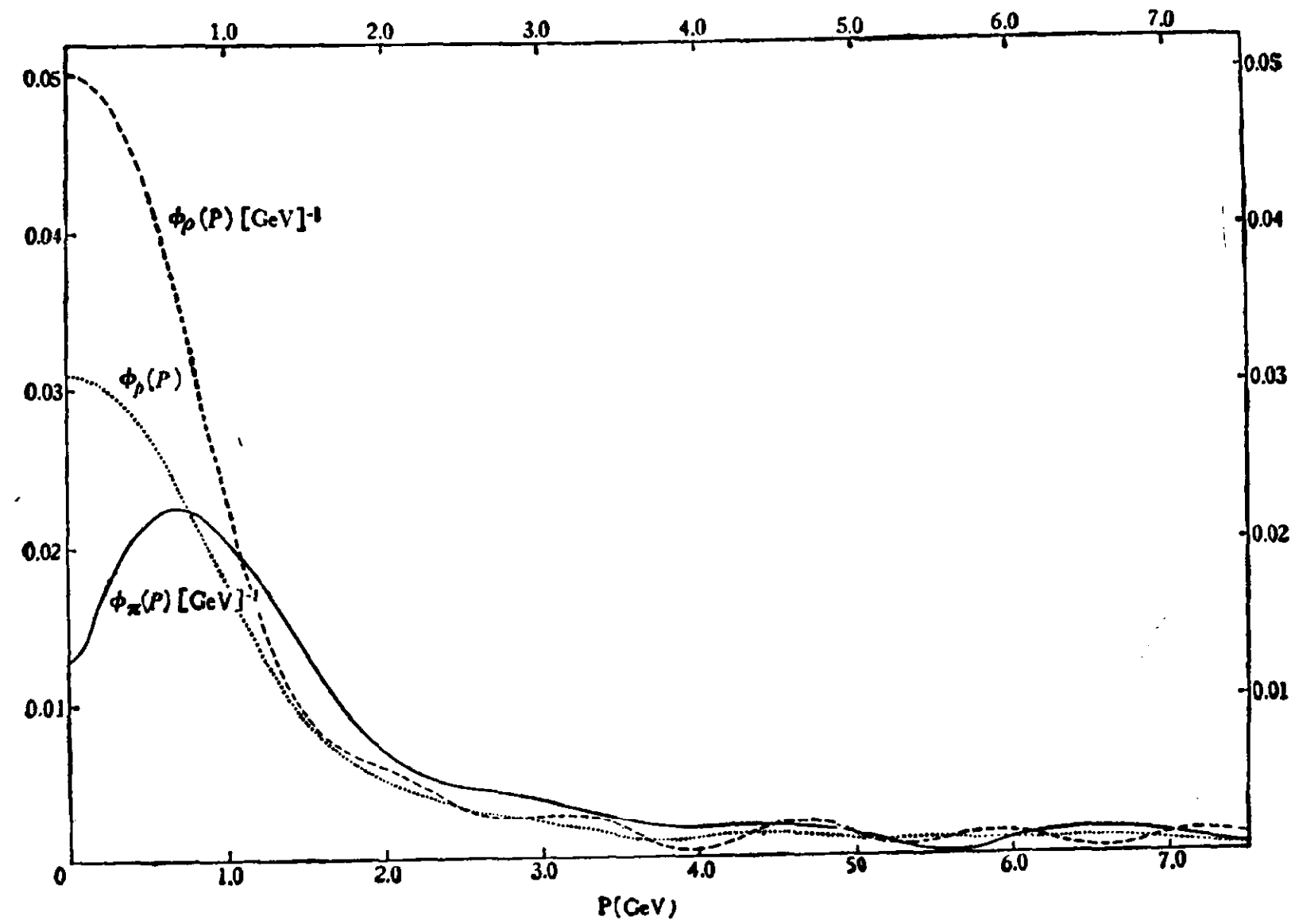

图 2

图 2.

由图 2 可见, $x$ 介子的波包函数与其它介子和重子的波包函数有明显区别,其原因在于 $x$ 介子是唯一的一个口袋半径小于康普顿波长的强子. 由此可以进一步看出, Donoghue 等人 ${ }^{[2,3]}$ 不加分析地将 $x$ 介子的 $\varphi(\boldsymbol{p})$ 推广到一切介子和重子的做法是不能令人满意的.

波包函数 $|\varphi(\boldsymbol{P})|$ 也可以利用近似公式 (10) 来计算. 在此情况下, 对于波包的中心高度 $\left|\varphi_{x}(0)\right|$, 得到

$$
\left|\varphi_{x}(0)\right|^{2}=\frac{2 m_{x}}{(2 x)^{6}} V_{x} .
$$

这正是在文献 [4] 中的公式 (16).

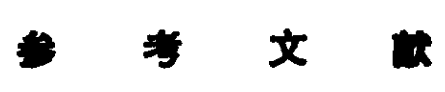

[ 1 ] Johneon, K., Phys. Lett., $78 B$ (1978), 259.

[ 2 ] Donoghue, J. F. Johnson, K., Phys. Rev., D21 (1980), 1975.

[ 3 ] Donoghue, J. F., Phys. Lett., 92B (1980), 99.

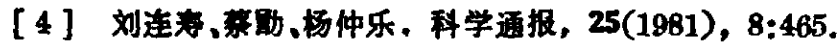

[5] Gundue, W., Hey, A. J. G. \& Walters, P. J., Phys. Rev., D21 (1980), 271.

[6] Schepkin, M., CERN Preprint. Ref. TH-2503, 1978.

[ 7 ] Din, A. M., Girardi, G. \& Sorba, P., LAPP-TH-08, 1979. 